\title{
Upper Gastrointestinal Endoscopy in ENT Practice: How Worth is It?
}

UP Santosh, MC Anup Kumar, Lavanya Karanam

\begin{abstract}
Objective: The need for upper gastrointestinal (GI) endoscopy in the evaluation of hoarseness.
\end{abstract}

Study design: Prospective study, conducted during the period from June 2012 to February 2013.

Setting: Tertiary referral center.

Results: A total of 125 patients were selected for the study and they were evaluated with appropriate history and clinical examination. Out of 125 patients, 41 (32.8\%) patients showed laryngeal findings leading to hoarseness, 13 (10.4\%) patients showed features of suspected malignancy in other adjacent regions which was confirmed later, two (1.6\%) patients showed phonetic gap and 69 (55.2\%) patients showed normal laryngeal inlet on indirect laryngoscopy examination. Of the 69 normal patients which were treated conservatively and since they did not show any response they were subjected to upper GI endoscopy. Out of 69 patients, $41(60 \%)$ patients showed features of gastritis, $28(40 \%)$ patients showed features of duodenitis.

Conclusion: It is estimated that more than $50 \%$ of patients presenting to the ENT OPD for hoarseness are because of $\mathrm{GI}$ problems. We strongly advise upper GI endoscopy for the symptomatic otorhinolaryngological patients with a normal laryngeal finding on indirect laryngoscopy for treating the condition accurately or near accurately. Adding to this upper $\mathrm{GI}$ endoscopy has the additional advantages of documentation and medicolegal aspect in the present day scenario.

Keywords: Hoarseness, Upper gastrointestinal endoscopy, Duodenitis.

How to cite this article: Santosh UP, Kumar MCA, Karanam L. Upper Gastrointestinal Endoscopy in ENT Practice: How Worth is It? Int J Phonosurg Laryngol 2013;3(2):35-38.

\section{Source of support: Nil}

Conflict of interest: None declared

\section{INTRODUCTION}

Hoarseness is one of the most commonly encountered symptoms in our clinical practice. It is the voice that is rough, grating, harsh more or less discordant and have lower pitch than normal for the individual. ${ }^{1}$ It is invariably the early manifestation of a large variety of conditions that directly or indirectly affect the voice apparatus.

In the words of Chevalier Jackson 'Hoarseness is a symptom of utmost significance and calls for a separate consideration as a subject because of its frequency as a distant signal of malignancy and other condition'. ${ }^{2}$

Its importance derives from the deplorable fact that though benign lesions are numerically more common cause of hoarseness than malignant diseases, opportunity of the cure has often been lost by delay under a benign diagnosis. ${ }^{2}$ Hoarseness is a common complaint in today's fast faced, high stressed life. ${ }^{3}$ Hence, there is a need for this study on hoarseness which is often treated inadvertently without getting into the details of the root cause.

\section{MATERIALS AND METHODS}

A total of 125 patients presenting to the ENT OPD, between June 2012 and February 2013 with complaints of hoarseness were selected for the study mainly based on history and clinical examination.

\section{Inclusion Criteria}

- All patients with hoarseness.

\section{Exclusion Criteria}

- Other voice disorders like rhinolalia clausa, rhinolalia aperta, articulation disorders and CNS and causes like bulbar palsy, Wegener's granulomatosis, MS, stroke and Parkinson's disease were excluded.

Hoarseness was evaluated for all patients. Appropriate history and clinical examination was done for all the patients with definite symptomatology. Then these patients were categorized into four groups based on the findings of indirect laryngoscopy (Graph 1).

First group (Figs 1A to D); 41 patients (32.8\%) having laryngeal findings:

- Interarytenoid mamilations

- Vocal cord nodules

- Vocal cord polyps

- Vocal cord paresis

- Vocal cord palsy

- Vocal cord growth

- False vocal cord HT, edema, thickening

- Myocarditis

- Diffuse laryngitis.

Second group (Figs 2A and B); 13 patients (10.4\%) with suspected malignancy, which was later confirmed on biopsy in the following regions:

- Post cricoid

- Base of tongue

- Epiglottis

- Pyriform fossa 


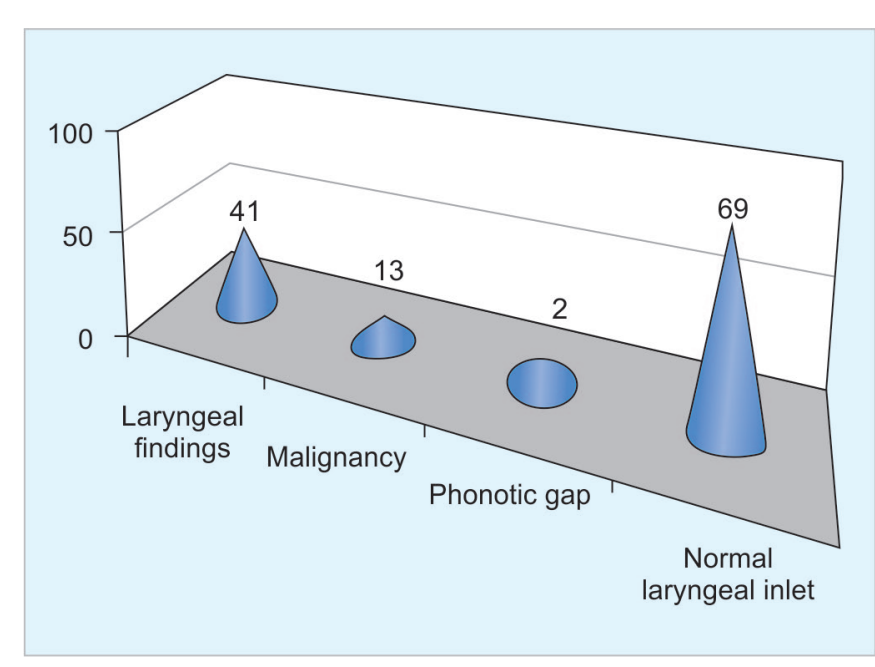

Graph 1: The distribution of groups

Third group (Fig. 3); two patients (1.6\%) with phonetic gap for which no cause was found when evaluated.

Fourth group (Fig. 4); 69 patients (55.2\%) with normal laryngeal findings.

Based on the laryngeal findings the first and second groups were treated accordingly. The third group was treated with reassurance, whereas the fourth group were subjected to upper gastrointestinal (GI) endoscopy for further evaluation of cause of hoarseness since the symptoms persisted for $>3$ weeks even after empirical treatment and reassurance.

Our endoscopic station was equipped with a Pentex EG291C camera, EPK150C light source and a $0.9 \mathrm{~cm}$ diameter scope with an emergency backup facility and procedure was done under xylocaine viscous after completing the formalities.

Among the 69 patients, 41 patients $(60 \%)$ had features suggestive of gastritis and 28 patients $(40 \%)$ had features suggestive of duodenitis (Table 1).

For gastritis the patients were treated using proton pump inhibitors for 2 weeks and for duodenitis the treatment adopted was with $H$. pylori kit comprising a combination of drugs for 2 weeks. These patients were treated and followed up for 3 to 6 weeks after completion of treatment. They showed marked improvement in relation to their symptoms.

\section{DISCUSSION}

Laryngopharyngeal reflux (LPR) is a modern day 'great masquerade'. ${ }^{4}$ It is defined as damage to the laryngeal

\begin{tabular}{lccc}
\multicolumn{4}{c}{ Table 1: Our findings of fourth group } \\
\hline $\begin{array}{l}\text { Endoscopic findings } \\
\text { (Figs 5 and 6) }\end{array}$ & Indirect laryngoscopy & Upper Gl endoscopy & $p$-value \\
\hline Gastritis and duodenitis & $0 / 125$ & $69 / 125(55.2 \%)$ & $12.4 \%,<0.001 \mathrm{HS}$ \\
Gastritis & $0 / 125$ & $41 / 125(32.8 \%)$ & $7.8 \%,<0.001 \mathrm{HS}$ \\
Duodenitis & $0 / 125$ & $28 / 125(20 \%)$ & $5.59 \%,<0.001 \mathrm{HS}$ \\
\hline
\end{tabular}

HS: Highly significant
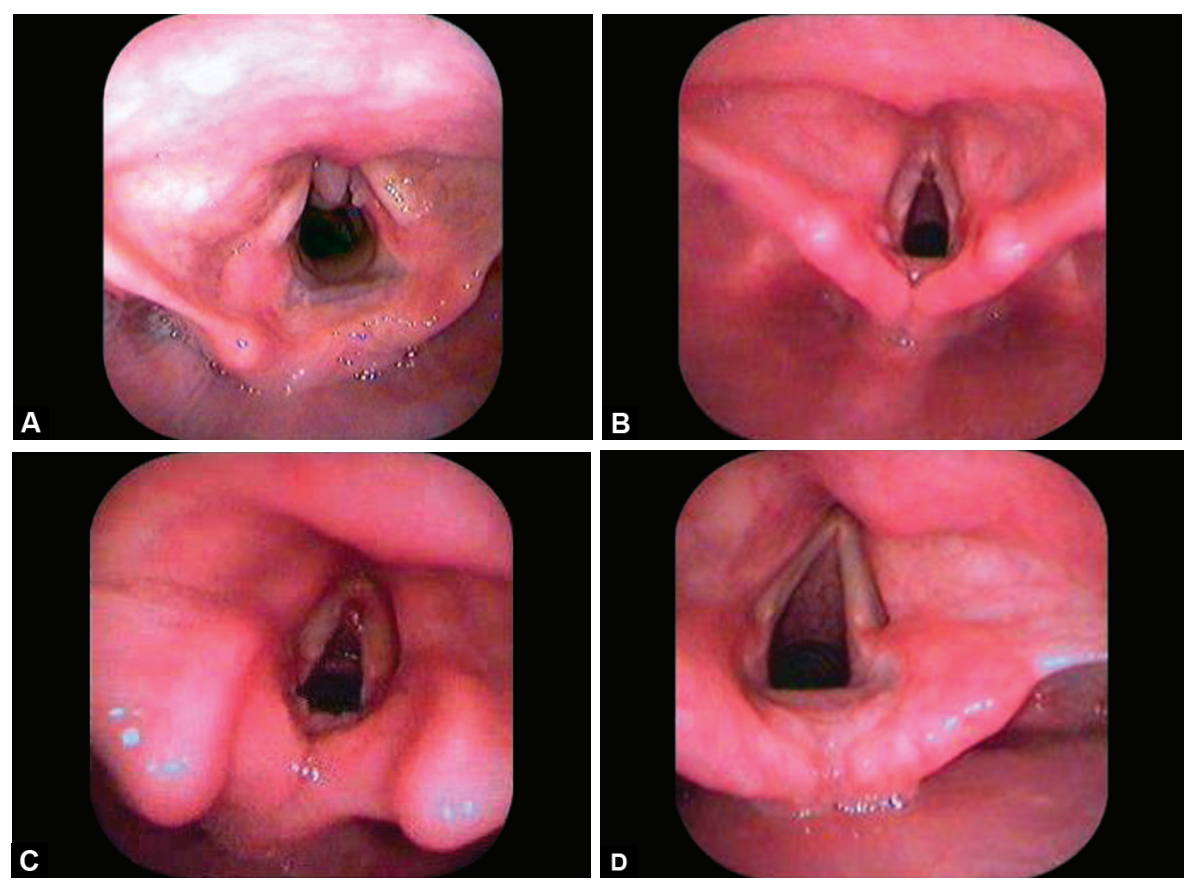

Figs 1A to D: (A) Anterior commisure growth with right vocal cord polyp, (B) bilateral vocal nodule, (C) interarytenoid mamilations, (D) left vocal cord palsy 

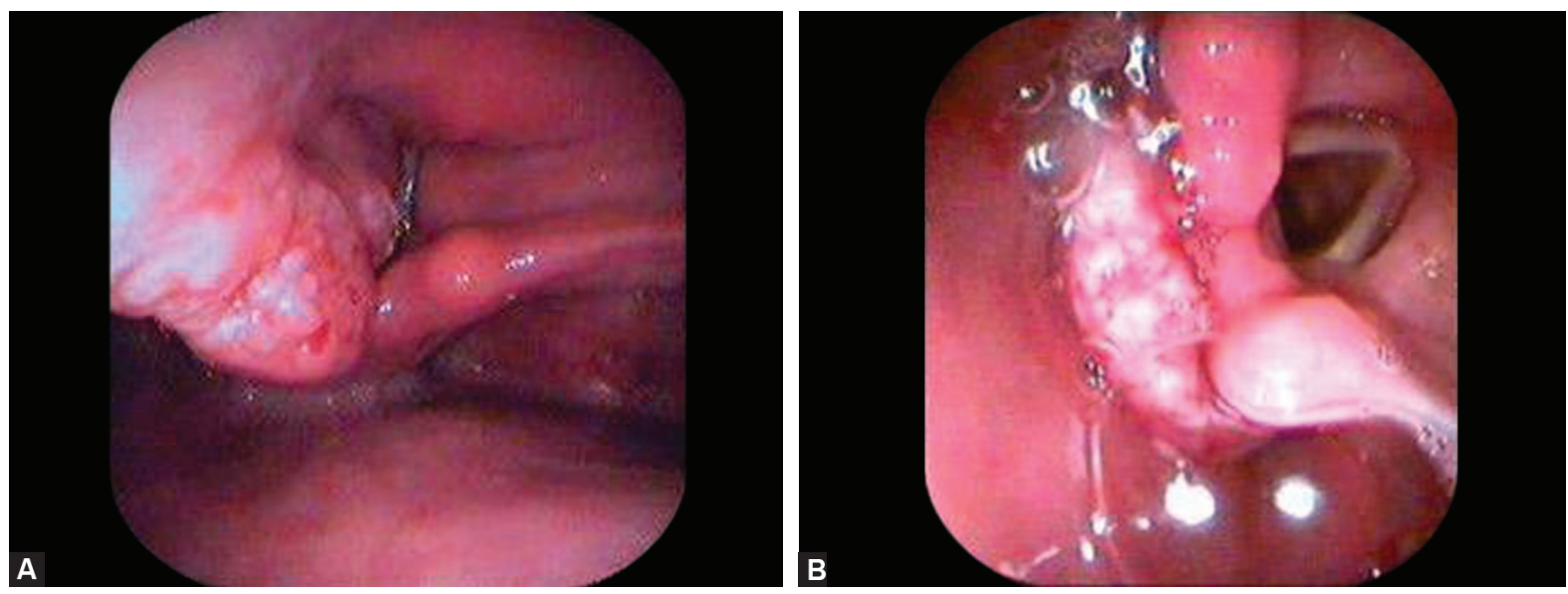

Figs 2A and B: (A) Pyriform fossa growth (left), (B) postcricoid malignancy

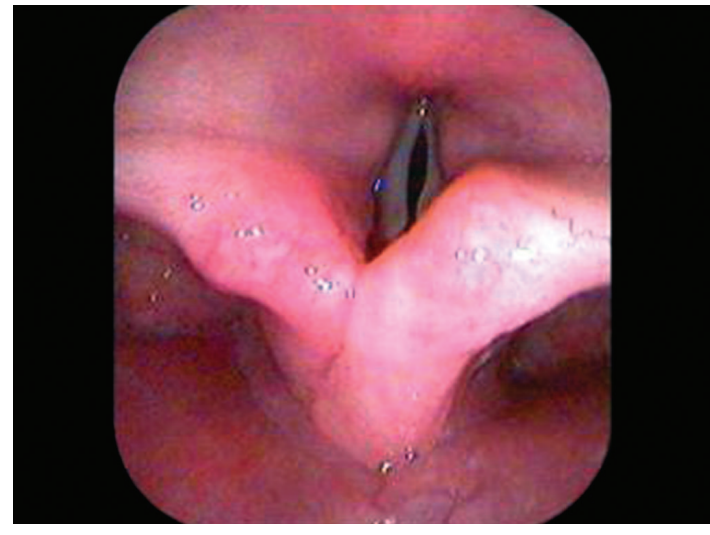

Fig. 3: Phonotic gap

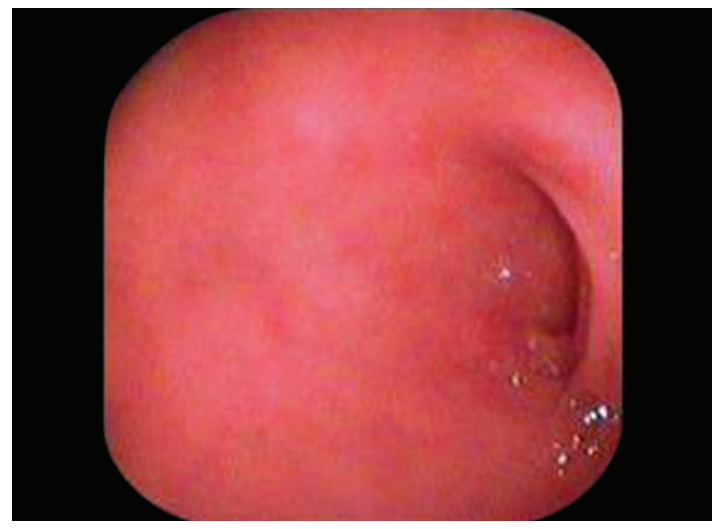

Fig. 5: Duodenitis

mucosa due to gastroduodenal contents either on a chronic or single incident. The past decade has witnessed increasing interest in the supraesophageal (ear nose, throat) manifestations potentially caused by gastroesophageal reflux disease (GERD). The availability of highly effective, medical and surgical themes allows for the control of these symptoms in many patients adding to the importance of accurately diagnosing LPR.

GERD is the most common malady of the esophagus. Complications of reflux disease involving structures proximally contiguous to the esophagus are now becoming

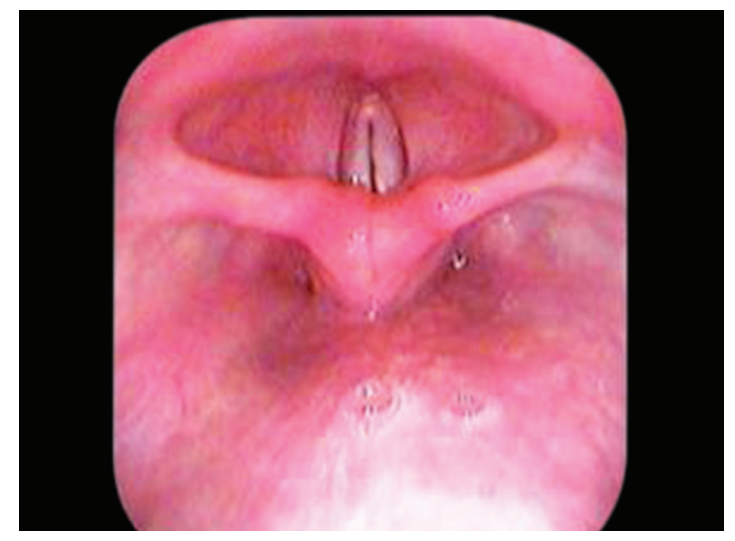

Fig. 4: Normal larynx

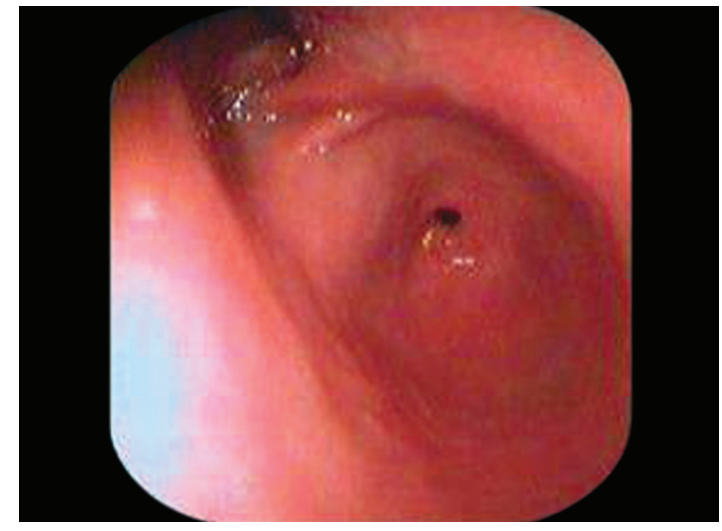

Fig. 6: Gastritis

a recognized clinical problem. There has been increasing interest in the possible relationship between laryngopharyngeal symptoms and GERD. ${ }^{5}$

Delahunty and Cherry ${ }^{6}$ were the first to describe GERD as a significant causative agent in the development of contact ulcers of the larynx. Gastroesophageal reflux occurs in 7 to $25 \%$ of the population on a daily or a monthly basis, respectively. ${ }^{7}$ It is estimated that 4 to $10 \%$ of patients presenting to ENT physicians do so because of complaints related to GERD. ${ }^{8}$ Hoarseness caused by GERD occurs in an estimated $10 \%$ of patients seen by ENT physicians. 
Studies, particularly that using 24-hour $\mathrm{pH}$ monitoring in patients with unresponsive hoarseness, find that 55 to $79 \%$ have acid reflux. ${ }^{9}$ GERD is the third leading cause of chronic cough, after sinus problems and asthma, accounting for $20 \%$ of cases. In these patients, either the degree of acid reflux is not well controlled on a day-to-day basis or other agents in the gastric refluxate, namely nonacidic duodenal contents (bile acids, pancreatic enzymes), are responsible for the ENT complaints and signs.

The specific agent(s) responsible for producing ENT symptoms and laryngeal pathology currently are unknown and the subject of many debates. ${ }^{6,10}$ Potential candidates include the gastric contents, acid and pepsin, and duodenal contents, both bile acids and the pancreatic enzyme trypsin. It is difficult to isolate the injurious potential of each of the earlier-listed agents because the gastric milieu refluxing into the esophagus is commonly a mixture of gastric and duodenal contents.

Furthermore, injury to the human larynx does not require large volumes or continuous reflux of acidic gastric contents, especially if antecedent injury has occurred to the vocal cord owing to viral infection, smoking, alcohol, or voice abuse. In theory, only intermittent exposure to small amounts of these injurious agents, as possibly seen in patients whose acid reflux is not well controlled, could cause laryngeal symptoms or injury. Therefore, pathophysiologically there are limited human data on the differential role of gastroduodenal contents in causing laryngeal injury or symptoms. However, recent animal experiments have helped to better clarify this important area of controversy. These experiments consistently show that pepsin and conjugated bile acid only in an acidic environment are the important injurious agents causing laryngeal inflammation.

Four animal studies have assessed the role of gastric contents in laryngeal pathology. ${ }^{6,10}$ In a study conducted by Koufman, ${ }^{10}$ acid exposure injured the subglottic region but most of the damage was seen with the combination of acid and pepsin. These observations are similar to animal studies in the esophagus finding that continuous esophageal exposure to acid alone at very low $\mathrm{pH}$ values or in combination with pepsin at $\mathrm{pH}$ values between 2 and 5 cause the greatest amount of mucosal damage. However, there are no human studies on this aspect till on date.

Being an otorhinolaryngologist, we stress on the importance of considering duodenitis as one of the upcoming and unknown cause for hoarseness, where indirect laryngoscopy is normal. Since, there are not much studies related to this entity this factor needs to be stressed upon.

\section{CONCLUSION}

It is estimated that more than $50 \%$ of patients presenting to the ENT OPD for hoarseness are because of GI problems. We strongly advise upper GI endoscopy for the symptomatic otorhinolaryngological patients with a normal laryngeal finding on indirect laryngoscopy for treating the condition with accuracy. Also upper GI endoscopy has the added advantages of documentation and medicolegal aspect in the present day scenario.

\section{REFERENCES}

1. Jackson, Chevalier L. Disease of ear, nose and throat. 2nd ed. Philadelphia: WB Saunders Company; 1959;576p.

2. Parik NP. Aetiology study of 100 cases of hoarseness of voice. Indian J Otolaryngol Head Neck Surg 1991Jun;43(2):71-73.

3. Batra K, Motwani G, Sagar PC. Functional voice disorders and their occurrence in 100 patients of hoarseness as seen on fibreoptic laryngoscopy. Indian J Otolaryngol Head Neck Surg 2004Apr;56(2):91-95.

4. Hawkins BL. Laryngopharyngeal reflux: a modern day 'great masquerade'. J Ky Med Assoc 1997Sep;95(9):379-385.

5. Ramdass T, Nithya N. Supraoesophageal manifestations of gerd - a myth or reality? Indian J Otolaryngol Head Neck Surg 2001April;53(2):168-172.

6. Delahunty JE, Cherry J. Experimentally produced vocal cord granulomas. Larygoscope 1986Nov;78(11):1941-1947.

7. Nebel OT, Fornes MF, Castell DO. Symptomatic gastroesophageal reflux: incidence and precipitating factors. Am J Dig Dis 1976 Nov;21(11):953-956.

8. Koufman JA, Wiener GJ, Wallace CW, Castell DO. Reflux laryngitis and its sequela. J Voice 1988;2:78-79.

9. McNally PR, Maydonovitch CL, Prosek RA, Collette RP, Wong RKH. Evaluation of gastroesophageal reflux as a cause of idiopathic hoarseness. Dig Dis Sci 1989 Dec;34(12):1900-1904.

10. Koufman JA. The otolaryngologic manifestation of gastroesophageal reflux disease (GERD): a clinical investigation of 225 patients using ambulatory 24-hour $\mathrm{pH}$ monitoring and an experimental investigation of the role of acid and pepsin in the development of laryngeal injury. Laryngoscope 1991 Apr;101 (4 Pt 2 Suppl 53):1-78.

\section{ABOUT THE AUTHORS}

\section{UP Santosh}

Professor, Department of ENT, JJM Medical College, Davangere Karnataka, India

\section{Anup Kumar}

Professor, Department of General Surgery, JJM Medical College Davangere, Karnataka, India

\section{Lavanya Karanam (Corresponding Author)}

Postgraduate Student, Department of ENT, JJM Medical College Davangere, Karnataka, India, e-mail: lavvi87@yahoo.com 